\title{
Major Depressive Disorder as a Post COVID Infection Sequela
}

\author{
Shahzaib Khan, Aaina Gupta, Palwasha Khan \\ School of Medicine, Ross University, Bridgetown, Barbados \\ Email: shahzaibkhan@mail.rossmed.edu
}

How to cite this paper: Khan, S., Gupta A. and Khan, P. (2021) Major Depressive Disorder as a Post COVID Infection Sequela. Open Journal of Internal Medicine, 11, 189-193.

https://doi.org/10.4236/ojim.2021.114014

Received: August 10, 2021

Accepted: October 18, 2021

Published: October 21, 2021

Copyright (อ 2021 by author(s) and Scientific Research Publishing Inc. This work is licensed under the Creative Commons Attribution International License (CC BY 4.0).

http://creativecommons.org/licenses/by/4.0/ (c) (i) Open Access

\begin{abstract}
COVID-19 has taken the world by storm, producing a multitude of concerning sequela in all genders and age groups. One surprising finding is the onset of depression post COVID recovery in patients with no past medical history of psychiatric illness. The following case report discusses a 52-year-old female with a past medical history of pneumonia secondary to COVID-19 in April 2021, who presented with recurrent falls and a syncopal episode. On review of systems, it was discovered that the patient has new onset depression since recovering from COVID related pneumonia. Patient has yet to follow up with an outpatient psychiatrist.
\end{abstract}

\section{Keywords}

Depression, Psychiatric Disorder, COVID-19, Pneumonia, Severe Acute Respiratory Syndrome Coronavirus 2, SARS-CoV-2

\section{Introduction}

Severe Acute Respiratory Syndrome Coronavirus 2 (SARS-CoV-2) is a single stranded RNA virus responsible for the Covid-19 pandemic. A study done in Wuhan, China showed that SARS-CoV-2 affected all patients from birth to those aged 70 and older [1]. Infections were uncommon between and including birth and adolescence whilst adults between the ages of 49 and 56 were the most susceptible with COVID-19 related hospitalizations increasing proportionally to age [1].

The average incubation period for the virus is two weeks and patients present with variable symptoms which can occur simultaneously or consecutively such as cough, being most prevalent, followed by fever, myalgias, headache, dyspnea, and loss of smell [2].

Recent studies demonstrate that COVID-19 patients with concomitant condi- 
tions such as Diabetes, Cardiovascular Disease and Chronic Obstructive Pulmonary Disease amongst others, undergo a more severe course of illness than their healthier counterparts [3] [4]. Onder G. et al. illustrated that COVID-19 patients have an average of three comorbidities [5].

Following initial infection, patients develop a multitude of sequelae ranging from acute respiratory failure and cardiac arrhythmias [6] to chronic respiratory symptoms [7] and continuous inflammatory responses [8] in multiple organ systems including myocardial inflammation [9]. A unique sequela of COVID-19 infection is an increase in new onset psychiatric illnesses in patients after hospital discharge. At one-month post-COVID-19 follow-up patients had an overall increase in Insomnia, PTSD, Anxiety and Depression [8]. A study done in 2009 by Sheng et al. reflected the findings when they reported an increase in psychiatric illness in patients after recovering from a coronavirus infection [10].

This case highlights the psychiatric consequences of COVID-19 after discharge from the hospital, with a focus on major depressive disorder.

\section{Case Presentation}

A 52-year-old female with a past medical history of COVID-19 infection (April 2021), menopause (2019), and hypertension presented with recurrent falls and a syncopal episode. According to the husband, she did not have any seizures. She said that she did not have any loss of urine or stool. There was no evidence of tongue biting. Lab workup was ordered which showed TSH and Vitamin B12 levels to be within normal limits. Syphilis was not detected, and her blood sugar was in the normal range. She had no history of any psychiatric illness.

After medication reconciliation, it was determined that the patient was taking both her new, $10 \mathrm{mg}$, and old, $5 \mathrm{mg}$ prescription of Amlodipine. Patient was admitted on the ward with administration of IV fluids as she was hypotensive. Her home medications were held. The patient was diagnosed with unintentional medication overdose.

After the patient achieved hemodynamic stability, she was educated on the importance of asking questions with regards to her health care and medications from her doctor and pharmacist. She was also advised to only take $10 \mathrm{mg}$ Amlodipine in the future and to follow up with her primary care physician.

Further questioning revealed mood changes post COVID-19 infection and hospitalization. Patient has subjective feeling of sadness and emptiness and a 20 lbs. weight loss associated with decreased appetite. Patient also has trouble sleeping. Before hospitalization, patient slept seven hours every night. Now, she sleeps for four hours with intermittent awakening throughout the night. Patient has been feeling fatigued, which she associates with poor sleep. She has lost interest in playing with her grandkids and has been unable to perform her daily tasks such as cooking and cleaning. Her husband has also noticed a lack of focus. Patient denies any suicidal ideation. She has been scheduled for follow up with an outpatient psychiatrist. 


\section{Discussion}

To diagnose Major Depressive Disorder, DSM-5 states that patients need to exhibit five or more symptoms within a two-week period, with one of the symptoms being a depressed mood or anhedonia [11]. This patient exhibited a 20 lbs. weight loss, anhedonia, depressed mood, loss of concentration, loss of appetite, insomnia, and fatigue nearly every day thereby fulfilling major depressive disorder criteria.

Apart from acute findings, COVID-19 infection can also cause a chronic inflammatory response leading to the production of cytokines, such as IFN Gamma, Interferon Beta, Interleukin four, six and ten [12]. Such chronic inflammation can damage the blood brain barrier allowing the cytokine storm to damage the integrity of the brain, further inciting dysregulation of neurotransmitters. This complex concoction of inflammatory mediators and their combined effect may explain the underlying mechanism of new onset psychiatric illness in COVID-19 patients post hospitalization [8].

The patient needs to be started on anti-depression medication such as a selective serotonin reuptake inhibitor (SSRI) to prevent further decline and progression to suicidal ideations. Patient also need to regularly follow up with an outpatient psychiatrist. Steroid therapy to control chronic inflammation should also be considered. There needs to be a re-emphasis on proper physician-patient communication to prevent medication induced hospitalization. The physician should take the time to address any medication changes in a way the patient easily understands, after which a two-way communication should ensure when the physician asks the patient if/she understood what was being said. Keeping this patient in mind, she is not focused and fatigued throughout the day which can alter her understanding of the information presented so extra care needs to be implemented with regards to her medication, for example, explaining her husband and asking him to make sure the correct medications are being taken. Writing down instructions on a piece of paper can also help.

This case also highlights the importance of correcting acute abnormalities followed by proper history taking with regards to psychiatric illnesses because many conditions can mimic psychiatric issues, however, ruling out common conditions and correcting acute abnormalities, true neuropsychiatric status can be assessed.

\section{Conclusions}

COVID-19 pandemic has proven itself to be a massive burden on all aspects of society producing worldwide shutdowns, major economic fluctuations, and monumental damage to the health of millions of people with repercussions to follow for many years to come. COVID-19 infection has a complex pathophysiology which explains its entanglement with all the major organs in the human body.

One such involvement is the neuropsychiatric domain which can be over- 
looked when compared to the more acutely visible and distressing symptoms seen in emergency departments around the world. COVID-19 related psychiatric illness has been shown to develop gradually upon discharge from the hospital perhaps due to failure of the inflammatory storm to suppress. Psychiatric illness, such as depression, should be on all healthcare providers' radar post COVID-19 infection and should be screened for as part of COVID-19 follow-up because if left untreated patients can develop a series of adverse symptoms, potentially culminating to suicide. Similarly, whenever a patient with a sudden, unexplained psychiatric illness presents itself, testing for prior COVID-19 infection should be undertaken.

\section{Conflicts of Interest}

The authors declare no conflicts of interest regarding the publication of this paper.

\section{References}

[1] Verity, R., Okell, L.C., Dorigatti, I., et al. (2020) Estimates of the Severity of Coronavirus Disease 2019: A Model-Based Analysis. The Lancet Infectious Diseases, 20, 669-677. https://doi.org/10.1016/S1473-3099(20)30243-7

[2] Stokes, E.K., Zambrano, L.D., Anderson, K.N., et al. (2020) Coronavirus Disease 2019 Case Surveillance, United States, January 22-May 30, 2020. Morbidity and Mortality Weekly Report, 69, 759-765. https://doi.org/10.15585/mmwr.mm6924e2

[3] Williamson, E.J., Walker, A.J., Bhaskaran, K., et al. (2020) Factors Associated with COVID-19-Related Death Using OpenSAFELY. Nature, 584, 430-436. https://doi.org/10.1038/s41586-020-2521-4

[4] CDC COVID-19 Response Team (2020) Preliminary Estimates of the Prevalence of Selected Underlying Health Conditions Among Patients with Coronavirus Disease 2019, United States, February 12-March 28, 2020. Morbidity and Mortality Weekly Report, 69, 382-386. https://doi.org/10.15585/mmwr.mm6913e2

[5] Onder, G., Rezza, G. and Brusaferro, S. (2020) Case-Fatality Rate and Characteristics of Patients Dying in Relation to COVID-19 in Italy. JAMA, 323, 1775-1776. https://doi.org/10.1001/jama.2020.4683

[6] Wang, D., Hu, B., Hu, C., et al. (2020) Clinical Characteristics of 138 Hospitalized Patients with 2019 Novel Coronavirus-Infected Pneumonia in Wuhan, China. JAMA, 323, 1061-1069. https://doi.org/10.1001/jama.2020.1585

[7] Van den Borst, B., Peters, J.B., Brink, M., et al. (2020) Comprehensive Health Assessment Three Months after Recovery from Acute COVID-19. Clinical Infectious Diseases, 73, e1089-e1098. https://doi.org/10.1093/cid/ciaa1750

[8] Mazza, M.G., De Lorenzo, R., Conte, C., et al. (2020) Anxiety and Depression in COVID-19 Survivors: Role of Inflammatory and Clinical Predictors. Brain, Behavior, and Immunity, 89, 594-600. https://doi.org/10.1016/i.bbi.2020.07.037

[9] Puntmann, V.O., Carerj, M.L., Wieters, I., et al. (2020) Outcomes of Cardiovascular Magnetic Resonance Imaging in Patients Recently Recovered from Coronavirus Disease 2019 (COVID-19). JAMA Cardiology, 5, 1265-1273. https://doi.org/10.1001/jamacardio.2020.3557

[10] Sheng, B., Cheng, S.K.W., Lau, K.K., Li, H.L. and Chan, E.L.Y. (2005) The Effects of 
Disease Severity, Use of Corticosteroids and Social Factors on Neuropsychiatric Complaints in Severe Acute Respiratory Syndrome (SARS) Patients at Acute and Convalescent Phases. European Psychiatry, 20, 236-242.

https://doi.org/10.1016/j.eurpsy.2004.06.023

[11] Park, L.T. and Zarate Jr., C.A. (2019) Depression in the Primary Care Setting. The New England Journal of Medicine, 380, 559-568.

https://doi.org/10.1056/NEJMcp1712493

[12] Ye, Q., Wang, B. and Mao, J. (2020) The Pathogenesis and Treatment of the "Cytokine Storm" in COVID-19. Journal of Infection, 80, 607-613.

https://doi.org/10.1016/j.jinf.2020.03.037 